\title{
Prevalence of peripheral neuropathy in diabetes mellitus and its association with therapy, ethnicity and duration of diabetes mellitus
}

\author{
Dipesh Karki ${ }^{1}$, Amar Nagila², Niraj Dhakal ${ }^{3}$, Sumit Chhetri ${ }^{4}$ \\ ${ }^{1}$ Consultant Physician, Department of Internal Medicine, Fishtail Hospital and Research Center, Pokhara, Nepal and \\ Lecturer, Department of Internal Medicine, Manipal College of Medical Sciences and Teaching Hospital, Pokhara, \\ Nepal, ${ }^{2}$ Professor, Department of Biochemistry, Gandaki Medical College Teaching Hospital and Research Center, \\ Lekhnath Pokhara, Nepal, ${ }^{3}$ Lecturer, Department of Biochemistry, Gandaki Medical College Teaching Hospital and \\ Research Center, Lekhnath Pokhara, Nepal, ${ }^{4}$ Department of Pharmacy, School of Health Sciences, Pokhara University, \\ Pokhara, Nepal
}

A B S T R A C T

Background: Diabetic peripheral neuropathy (DPN) is a most common micro vascular complication of diabetes posing significant morbidity and mortality with early and insidious onset. Studies has variably pointed that older age, gender, duration of diabetes, dyslipidemia, are linked with development of DPN. Aims and Objectives: To determine the prevalence of neuropathy in diabetic patients and see its link with age, gender, duration of diabetes, therapy and ethnicity. Materials and Methods: This is a cross-sectional study involving 110 clinically diagnosed diabetic patients, meeting the inclusion criteria. Basic demographic data were taken during clinical examination. Presence of degree of neuropathy was screened by measuring vibration perception threshold using Biothesiometer. Data was entered on SPSS and were categorized, necessary non parametric statistical tests were applied to these categorical variables as needed. The level of significance was set at $p$ value less than 0.05. Results: Presence of neuropathy was measured in 110 diabetic patients. The overall prevalence of DPN was $45.45 \%$. Frequency of neuropathy was higher with increase in age with $72.7 \%$ in more than 60 years of age and $23.5 \%$ in less than or equal to 40 years of age group $(p=0.007)$. Similarly, the increasing trend of DPN with increase in duration of DM was evident in this study with DPN present in more than $55 \%$ of patients with more than 5 years of diabetes $(p=0.004)$. Conclusion: The prevalence rate of DPN in diabetes was observed to be higher $(45.45 \%)$. Our result suggested that DPN is associated with old age and the duration of diabetes mellitus.

Key words: Biothesiometry; Diabetic polyneuropathy (DPN); Diabetes Mellitus(DM)
http://nepjol.info/index.php/AJMS DOI: 10.3126/ajms.v10i1.21743 E-ISSN: 2091-0576 P-ISSN: 2467-9100

\section{INTRODUCTION}

Diabetes mellitus (DM) is a chronic hyperglycemic condition due to metabolic derangements mainly attributed to absolute insulin deficiency or defect in insulin action. ${ }^{1,2}$ Almost $6 \%$ of world's population are affected by DM and the burden is increasing at an alarming rate. ${ }^{2} \mathrm{Nepal}$ is also facing the increase burden of DM as like other nations globally. ${ }^{2}$ It is assumed that one in ten Nepalese have DM and the prevalence is higherin compare to India, Pakistan and Bangladesh..$^{3-6}$ Studies from Nepal further indicates that about $50 \%$ of the cases of DM remain undiagnosed. ${ }^{5}$ Compounded by lack of quality health service, lack of routine health checkup, personal ignorance, and late consultation to health care provider, the complications of DM might be higher or more severe in our setting. ${ }^{2}$ 
Diabetes mellitus poses significant morbidity and mortality if untreated because of its associated late complications in the form of microvascular and macro-vascular structures. ${ }^{1}$ Complications are imputed to hyperglycemia, non-enzymatic glycation, oxidative stress, dyslipidemia, increased polyol pathway activity, altered perfusion, etc. that are seen in diabetic patients. ${ }^{1}$ Development of diabetic complications and its severity is correlated to length of DM. ${ }^{5}$ Furthermore, people with type-2 DM are at high risk of complications due to its insidious nature and late diagnosis, than type -1 DM. ${ }^{2}$

Neuropathy has been considered as important micro vascular complication of diabetes and sometimes reasoned as the pathological process for other abnormalities in DM. 1,7,8 Neuropathy is present from early stage of DM which slowly progresses over several years causing systemic damage. ${ }^{1}$ Diabetic wound classification system proposes that the risk of amputation increases by 1.7 times in case of diabetic neuropathy. ${ }^{7,9}$ Foot ulcers, charcots joints, numbness, paresthesia, loss of coordination, burning, pricking pain, abnormal ankle reflexes, etc. are manifestations of neuropathy. ${ }^{1}$ Studies shows about $50-90 \%$ of diabetics sooner or later develop one or other types of neuropathy. ${ }^{1,10}$ The prevalence of diabetic neuropathy ranges from $9.6 \%$ to $78 \%$ in different populations making it the most frequent chronic complications of DM. ${ }^{1,11,12}$ Study from Nepal has also reported the high prevalence of diabetic neuropathy correlating with older age and long duration DM to be significant risk factors. ${ }^{10,13,14}$

About $60-70 \%$ of foot ulcer that appear in diabetic patients are primarily neuropathic in origin. ${ }^{12}$ The vicious cycle which involves chronic hyperglycemia, spontaneous glycation of proteins, defect in nerves, metabolic derangements, alterations of micro-vessels, hypoxia, ischemia, inflammation, etc. are implicated in developing neuropathy. ${ }^{111}$ Development of neuropathy and its severity increases with increase in duration of DM and poor glycemic control'; a study shows, there is $49 \%$ chance of getting neuropathy with 25 years old diabetic history. ${ }^{5,15}$ Similarly in another study, strict glycemic control by intensive therapy was found to significantly reduce microvascular complications like neuropathy but the findings are still contradictory. ${ }^{7}$ Many studies have found ethnic variation in diabetic neuropathy. A study done in U.K reports lower incidence of neuropathy in Asians than White population, but the outcomes are disparate with no explanations. ${ }^{16}$ However, studies variably indicates that older age, gender, duration of DM, dyslipidemia, ethnicity are associated with diabetic neuropathy, so, much in depth studies are needed to explore the reasonable explanation. ${ }^{12}$ Since diabetes has higher prevalence in South Asian region with poor and low income countries experiencing huge burden. ${ }^{17}$ Thus, it is logical to speculate high rate of diabetic complications like neuropathy, in people living in these areas.

Neuropathy is most common complication of DM which starts subtly. A better understanding of this progressive condition is essential for better prognosis. ${ }^{1}$ Early identification and suitable management of neuropathy in diabetic subjects is essential for many reasons: 1) many therapeutic options are available for symptomatic patients; 2) about $50 \%$ of neuropathy can be asymptomatic and patient are at risk of insensate foot injury; 3) reduce the foot ulceration and amputation. ${ }^{5}$ Nepal is low income country in south Asian region and thus lies in high prevalence zone for diabetes, and thus, likely to have many diabetic people with neuropathies and other chronic complications. Limited studies have been done about chronic diabetic complications like neuropathy, which necessitates the requirement of further research in our setting to make nationwide database. Moreover, there was no published data to evaluate DPN by using the biothesiometry. Therefore, in this study we have made effort to find the prevalence of diabetic neuropathy using biothesiometry. Furthermore, we investigated the association of DPN with ethnicity, duration of DM and diabetic therapy.

\section{MATERIALS AND METHODS}

This is a Hospital based cross-sectional study conducted in Fishtail Hospital and Research Center Pvt. Ltd. One hundred and ten clinically diagnosed diabetic patients attending the Department of Internal Medicine, Fishtail Hospital\& Research Center, who were under anti-diabetic therapy, were enrolled. Ethnical Clearance was sought from the Research Committee of Fishtail Hospital\& Research Center and verbal consent was taken from all the study subjects during clinical examination.

Detailed history and clinical examinations were carried out in enrolled subjects. They were then subjected to neuropathy screening using biothesiometry which is sensitive method to detect and evaluate peripheral neuropathy with impaired vibratory perception threshold (VPT), mainly in diabetology and neurology. Its principle is vibrating probe, where amplitude of vibration can be altered by adjustment of voltage. ${ }^{13,18}$ Vibration perception threshold volts was measured at six different sites of the sole (great toe, $1^{\text {st }}$ metatarsal, $3^{\text {rd }}$ metatarsal, $5^{\text {th }}$ metatarsal, Instep and Heel) of each foot using biothesiometer and mean value was recorded. The mean value of $\leq 15$ volts indicates normal; $15-20$ volts indicates mild loss of VPT; $20-25$ volts indicates moderate loss of VPT and $>25$ volts indicates severe loss of VPT (Foot is at high risk). ${ }^{13} \mathrm{VPT}$ 
was measured using Biothesiometer and the data were categorized in different age groups, medication groups (monotherapy and polytherapy), ethnicity and duration of DM. Data was entered in SPSS version 20 and variables were categorized in different headings (age group, duration of DM, ethnicity, therapeutic mode, gender, etc.). Descriptive statistics were used to identify DPN prevalence, mean, and standard deviation. Chi square test was applied to see the association between categorical variables. Statistical significance was set at $\mathrm{P}<0.05$.

\section{RESULT}

One hundred and ten diabetic patients were studied for the presence of diabetic peripheral neuropathy (DPN). The overall basic and clinical characteristics of study subjects are shown in Table 1.

There were 45 female (41\%) and 65 male (59\%) with the mean age of $54.55 \pm 12.52$ years ranging from 30 to 92 years; most of the patients were more than in their fifties. The overall prevalence of DPN was found to be $45.45 \%$. In male the prevalence of DNP was $47.7 \%$ whereas the prevalence rate in female was $42.2 \%$. In both gender mild neuropathy was most common complaint and severe form was present in only $4.5 \%$ of the patients. However, more than half of the diabetic patients i.e. $60(54.5 \%)$ had no symptoms neuropathy. The association between gender and neuropathy was not significant $(p=0.22)$

As shown in figure 1, the prevalence of DPN was higher with increase in age, from $23.5 \%$ in $\leq 40$ years age group; $36.7 \%$ in age group $41-60$ years and $72.7 \%$ in $>60$ years age group, Furthermore, the severe neuropathy was also present in older age. Presence of neuropathy with increasing age was highly significant $(p=0.007)$. Similarly, comparison between ethnicity showed more Brahmins with neuropathy than other ethnic groups, however, the ethnic variation was not significant $(p=0.12)$

In the meantime, the association between DPN and duration was DM was highly significant $(p=0.004)$ as shown in figure 2. The rate of DPN was higher with increase in duration of DM, $31.0 \%$ in $<5$ years, $58.8 \%$ in $5-10$ years and $66.6 \%$ in $>10$ years with severe neuropathy seen above 5 years $(4.5 \%)$ of DM.

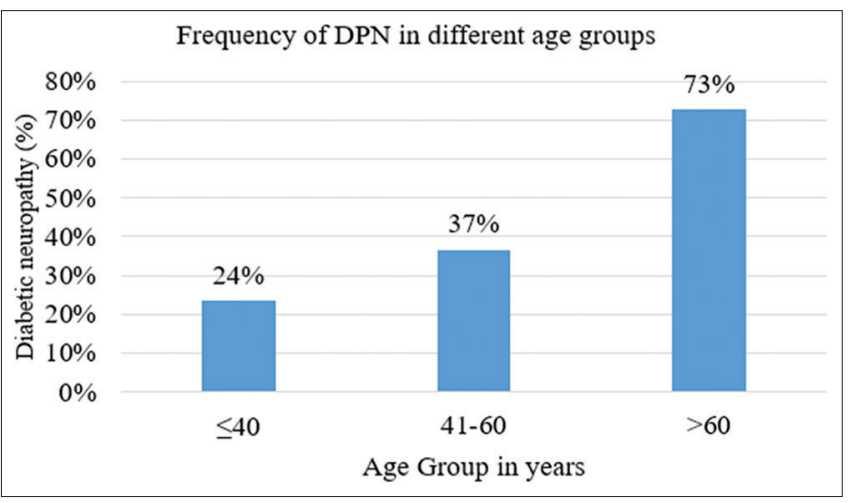

Figure 1: Prevalence of peripheral neuropathy in diabetic patients in different age groups

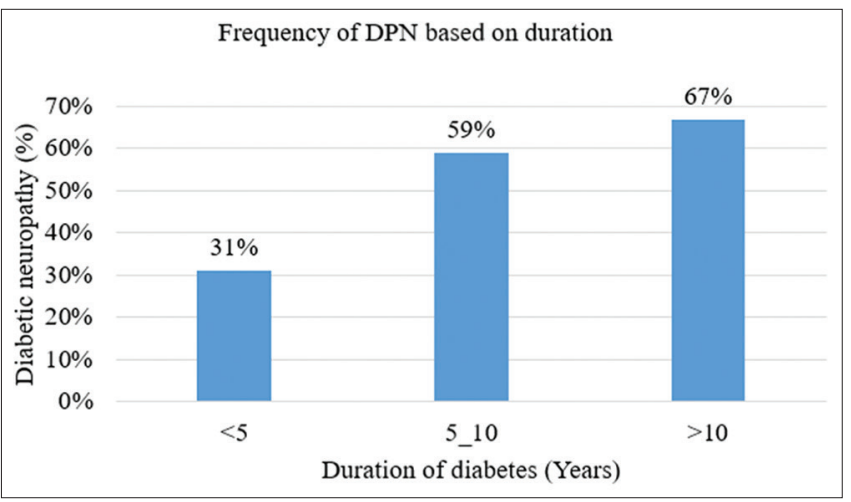

Figure 2: Prevalence of peripheral neuropathy in diabetic patients according to duration of diabetes mellitus

\section{Table 1: General and clinical characters of diabetes mellitus patients}

\begin{tabular}{|c|c|c|c|c|c|}
\hline \multirow[t]{2}{*}{ Variables } & \multicolumn{4}{|c|}{ Neuropathy } & \multirow[t]{2}{*}{$\chi^{2}$ test $P$ value } \\
\hline & Normal $\mathrm{N}=60 \mathrm{~N}(\%)$ & Mild N=34 N (\%) & Moderate $\mathrm{N}=11 \mathrm{~N}(\%)$ & Severe $N=5 \mathrm{~N}(\%)$ & \\
\hline \multicolumn{6}{|l|}{ Gender } \\
\hline Male & $34(52.3)$ & $24(36.9)$ & $4(6.2)$ & $3(4.6)$ & 0.22 \\
\hline Female & $26(57.8)$ & $10(22.2)$ & $7(15.6)$ & $2(4.4)$ & \\
\hline \multicolumn{6}{|l|}{ Age (Years) } \\
\hline$\leq 40$ & $13(76.5)$ & $4(23.5)$ & $0(0)$ & $0(0)$ & 0.007 \\
\hline $41-60$ & $38(63.3)$ & $16(26.7)$ & $4(6.7)$ & $2(3.3)$ & \\
\hline$>60$ & $9(27.3)$ & $14(42.4)$ & $7(21.2)$ & $3(9.1)$ & \\
\hline \multicolumn{6}{|c|}{ Duration of DM (Years) } \\
\hline$<5$ & $40(69.0)$ & $16(27.6)$ & $2(3.4)$ & $0(0.0)$ & 0.004 \\
\hline $5-10$ & $14(41.2)$ & $14(41.2)$ & $3(8.8)$ & $3(8.8)$ & \\
\hline$>10$ & $6(33.3)$ & $4(22.2)$ & $6(33.3)$ & 2 (11.1) & \\
\hline \multicolumn{6}{|c|}{ Therapeutic Mode } \\
\hline Monotherapy & $38(59.4)$ & $20(31.2)$ & $4(6.2)$ & $2(3.1)$ & 0.32 \\
\hline Polytherapy & $22(47.8)$ & $14(30.4)$ & $7(15.2)$ & $3(6.5)$ & \\
\hline
\end{tabular}

$\mathrm{N}$ stands for number 
All the patients were under medication, either monotherapy or polytherapy. However, neuropathy was present in these patients with $52.1 \%$ under polytherapy and $40.6 \%$ under monotheraphy had DPN, but majority of patients $(54.5 \%)$ under medication had normal finding. Although, this was found to be insignificant $(p=0.32$ ).

\section{DISCUSSION}

The prevalence of DPN in our study was found to be $45.45 \%$ with male and female equally affected which is comparable with study done by Yogi et al. ${ }^{1}$ and Dyck et al. ${ }^{8}$ which has reported the prevalence of $48 \%$ and $47 \%$ respectively. ${ }^{1}$ The study done by Karki et al. ${ }^{13}$ in Kathmandu Medical College has reported higher prevalence rate of $58.7 \%$ and similarly a European study ${ }^{19}$ also reported overall DPN prevalence of $60 \%$ which was higher than our study. However, several other studies done in different places has reported low prevalence rate of DPN in DM $\left(19.7 \%,{ }^{20} 19.1 \%,{ }^{21}\right)$. In our study there was no significant difference in DPN between male and female which is in accordance with other studies. ${ }^{13,}{ }^{22}$ Although studies have reported conflicting results, the effect of gender on neuropathy remains elusive. ${ }^{23}$ Study from Nepal reported the high prevalence of diabetic neuropathy ranging from $22.2 \%$ to $58.7 \%$ in different regions, ${ }^{10,13,14}$ correlating with older age and long duration of DM to be significant risk factors for neuropathy ${ }^{13}$, similarly, another study showed the prevalence of $44.4 \% .^{10}$

There was highly significant difference in prevalence DPN in different age groups and duration of DM; DPN was more prevalent with increase in age and long duration of DM. In accordance with our findings numerous studies has shown that increasing age and long standing DM are significant risk factors of DPN in DM. ${ }^{7,13,21}$ Although not significant in this study, therapy did not significantly reduce the burden neuropathy which is in accordance with other literatures. ${ }^{23,24}$ However, it is widely agreed that controlling hyperglycemia is the only option to reduce the onset and progression of neuropathy while no treatment options are available. ${ }^{7}$ A study reasons that frequency of occurrence of neuropathy in DM patients under therapy depend on type of DM, duration of treatment and duration of DM. ${ }^{23}$

\section{LIMITATIONS OF THE STUDY}

Only biothesiometry was used and it could not be compared with nerve conduction study which is a gold standard for diagnosing peripheral neuropathy. In addition, we were unable to rule out other confounding factors which could affect the results.

\section{CONCLUSION}

Peripheral Neuropathy was measured by using biothesiometry which is based on assessment of Vibratory perception threshold measurement. It is easy, cheap and precise tool for detecting peripheral neuropathy and widely used by clinicians in diabetology or neurology practice. It can even be used in field studies for screening purpose. Chronic DM is commonly associated with DPN which is evident from our study with high prevalence rate of $45.45 \%$. Further, the prevalence rate increased with duration of DM and increasing age. In addition, although intensive therapy to control hyperglycemia can reduce incidence of DPN. However, in this study the prevalence of DPN in DM patients was not significantly different among monotherapy or polytherapy groups.

\section{ACKNOWLEDGEMENTS}

We highly acknowledge the support of Fishtail Hospital and Research Center where this study was done. We acknowledge all the subjects without whose involvement this study would not be possible.

\section{REFERENCES}

1. Yogi K. Micro-vascular complications in diabetic patient's of type2 diabetes mellitus. Journal of Institute of Medicine 2009;31(1).

2. Singh NK, Shah NK, Bhandari A, Pandey S and Sharma SJ. Presentations and complications of diabetes patients presenting to diabetic clinic of Eastern Nepal. Journal of College of Medical Sciences-Nepal 2014; 9(3):25-30.

3. Maskey R, Shakya D, Sharma S, Karki P and Lavaju P. Diabetes mellitus related complications in out-patient clinic of tertiary care hospital. Journal of College of Medical Sciences-Nepal 2011; 7(2):9-16.

4. Singh Dand Bhattarai M. High prevalence of diabetes and impaired fasting glycaemia in urban Nepal. Diabetic medicine 2003; 20(2):170-171.

5. Dulal RK and Karki S. Disease management programme for diabetes mellitus in Nepal. Journal of the Nepal Medical Association 2009;48(176):281-286.

6. White $F$ and Rafique $G$. Diabetes prevalence and projections in South Asia. The Lancet 2002;360(9335):804-805.

7. Nisar MU, Asad A, Waqas A, Ali N, Nisar A, Qayyum MA, et al. Association of diabetic neuropathy with duration of type 2 diabetes and glycemic control. Cureus 2015;7(8).

8. Dyck PJ, Kratz K, Karnes J, Litchy WJ, Klein R, Pach J, et al. The prevalence by staged severity of various types of diabetic neuropathy, retinopathy, and nephropathy in a population based cohort: the RochesterDiabetic Neuropathy Study. Neurology 1993; 43(4):817-824.

9. Armstrong DG, Lavery LA and Harkless LB. Validation of a diabetic wound classification system: the contribution of depth, infection, and ischemia to risk of amputation. Diabetes care 1998; 21(5):855-859.

10. Almuhannadi $\mathrm{H}$, Ponirakis $\mathrm{G}$, Khan A and Malik RA. Diabetic 
neuropathy and painful diabetic neuropathy: cinderella complications in South EastAsia. J Pak Med Assoc 2018;68:85-89.

11. Emanuel AL, Nieuwenhoff MD, Klaassen ES, Verma A, Kramer MH, Strijers R, et al. Relationships between type 2 diabetes, neuropathy, and microvascular dysfunction: evidence from patients with cryptogenic axonal polyneuropathy. Diabetes Care 2017:dc161690.

12. Shrestha $\mathrm{H}$ and Katwal P. Prevalence and Risk Factors of Diabetic Peripheral Neuropathy in T2DM Patient Presenting to Commnity Hospital in Nepal. Kathmandu Univ Med J 2017; 58(2):146-149.

13. Karki D, Yadava S, Pant S, Thusa N, Dangol E and Ghimire S. Prevalence of sensory neuropathy in type 2 diabetes mellitus and its correlation with duration of disease. Kathmandu Univ Med J (KUMJ) 2016;14(54):120-124.

14. Rimal A and Panza A. Prevalence of, and factors associated with, type 2 diabetes and its microvascular complications among the elderly in Kathmandu, Nepal. J Health Res 2013; 27(1).

15. Organization WH. Definition, diagnosis and classification of diabetes mellitus and its complications: report of a WHO consultation. Part 1, Diagnosis and classification of diabetes mellitus. Geneva: World health organization, 1999.

16. Boulton A. The pathogenesis of diabetic foot problems: an overview. Diabetic Med. 1996;13:S12-S16.
17. Forouhi NG and Wareham NJ. Epidemiology of diabetes. Medicine 2010;38(11):602-606.

18. Kasalova Z. Biothesiometry in the diagnosis of peripheral neuropathies. Casopis lekaru ceskych 2002;141(7):223-225.

19. Boru TU AR, Sargin $H$, Kouer A, Sargin $M$ and Luleci $A$. Prevalence of peripheral neuropathy in type 2 diabetic patients attending a diabetes center in Turkey. Endocrinology Journal 2004;51:563-567.

20. Mrokrid K AL and Hussain A. Risk factors and prevalence of diabetic peirpheral neuropathy: a study of type 2 diabetic outpatients in Bangladesh. International Journal of Diabetes Dev Ctries 2010;30:11-17.

21. Ashok S RM, Deepa R and Mohan V. Prevalence of neuropathy in type 2 diabetic patients attending a diabetes center in South India. Journal of Associated Physicians India 2002;50:546-550.

22. Janghorbani $M$ RH, Kachooei $A$, Ghorbani $A$, Chitsaz $A$, Izadi $F$, et al. Peripheral neuropathy in type 2 diabetes mellitus in Isfahan Iran: Prevalence and risk factors. Acta Neurol Scand 2006; 114:384-391.

23. Valensi P, Giroux C, Seeboth-Ghalayini B and Attali J-R. Diabetic peripheral neuropathy: effects of age, duration of diabetes, glycemic control, and vascular factors. Journal of diabetes and its complications 1997;11(1):27-34.

24. Tesfaye S. Neuropathy in diabetes. Medicine 2010;38(12):649-55.

\section{Authors Contribution:}

DK- Brought the concept of the study and designed it and involved in critical revision; AN- Concept and design of the study as well as critical revision;

ND- Statistical analysis and manuscript preparation; SC- Literature review and manuscript preparation.

Work attributed to: Fishtail Hospital and Research Center.

Orcid ID:

Dr. Dipesh Karki- (i) https://orcid.org/0000-0002-4789-8765

Dr. Amar Nagila- (1) https/orcid.org/0000-0002-4001-5122

Mr. Niraj Dhakal- (1) https://orcid.org/0000-0002-9299-0501

Source of Support: Since this is the study done in outpatients visiting the clinic, all the tests are done as per routine checkup. All the requirements are provided by the research committee of Fishtail Hospital and Research Center. Conflict of Interest: None. 